\title{
ASPECTS OF AVERAGE RESPONSE COMPUTATION BY APERIODIC STIMULATION*
}

\author{
M. TEN HOOPEN \\ Institute of Medical Physics, TNO, Utrecht, Netherlands \\ and \\ H. A. REUVER \\ Technological University of Twente, Enschede, Netherlands
}

\begin{abstract}
A mathematical analysis of the variance of the average evoked-response computation as a function of the number $N$ of stimuli presented is made for the case when the response is disturbed by additive stationary noise. A comparison is made between the variance for purely periodic stimuli and that for stimuli of which the interstimulus durations are Gaussian distributed. In the latter situation, the interval durations may be correlated with each other, e.g. according to a Gaussian Markov process. It is deduced that, in general, the introduction of aperiodic stimulation tends to make the functional relationship between the variance and $N$ behave as though it holds for noise with a very broad frequency spectrum; the variance is proportional to $1 / N$.
\end{abstract}

\section{INTRODUCTION}

IT IS well known that, for example in the field of electroencephalographic (e.e.g.) analysis, the quality of a repeatedly evoked response that is disturbed by noise may be improved by a procedure of summation and averaging. If the response is deterministic, on summation, its amplitude increases linearly with the number of stimulations, $N$, whereas, as a rule of thumb, the standard deviation or r.m.s. value of the ampliude of the noise increases with $\sqrt{ } N$, so that the signal/noise ratio expressed in terms of the amplitude increases with $\sqrt{ } N$, or, in power terms, the signal/noise ratio increases with $N$. Several conditions must, however, be fulfilled for this simple rule to hold true exactly. The duration of the evoked potential must be smaller than the interstimulus duration $m$. The noise must be additive to the response, not time-locked to the response, and it must constitute a stationary random process. Moreover, in the case of regular stimulation, the period $m$ must be such that, after a time greater than $m$, the noise is not correlated with itself. If the noise has a more or less narrowband power spectrum, the successive samples of the noise may be correlated with each other, and the signal/noise ratio in power terms may be larger or smaller than $N$. If, in studies of evoked response, the alpha rhythm represents the noise component, the assumption of independence of the noise samples is in general not justified.

LEE (1960) has indicated that the correlation between the sample amplitudes of a stationary random process can be diminished by taking the samples not in a purely regular fashion, but by introducing fluctuations in the time durations between samples. RUCHLIN (1965) has worked out the idea of improving average-response computation by diminishing the correlation between subsequent noise-sample amplitudes; he suggested the use of aperiodic stimulation. Ruchlin investigated the effect for stimulusinterval durations which were equiprobably distributed between two values, $m-m_{a}$ and $m+m_{a}$, or exponentially distributed with a dead time $m_{c}$ and mean $m$, as visualised Figs. $1 a$ and $b$. It will be noted that the observation 
time for the evoked response is diminished from $m$ for regular stimulation to, respectively, $m-m_{a}$ and $m-m_{c}$ for the irregular stimulus pattern, when the mean interval duration is the same and equal to $m$. As to the noise, Ruchlin's computation was carried out for a flat power spectrum of bandwidth $f_{x}$ and centre frequency $f_{c}$.
As will be shown, for a given noise spectrum with centre frequency $f_{c}$, the deviation from the law $\operatorname{var} N=1 / N$, where $\operatorname{var} N$ denotes the normalised variance of the sample mean, is most pronounced if the value of $f_{c}$ is equal to, or nearly equal to, half the stimulus frequency $f_{s}(=1 / m)$ or to multiples thereof; thus for $f_{c}=f_{s} / 2, f_{c}=f_{s}$
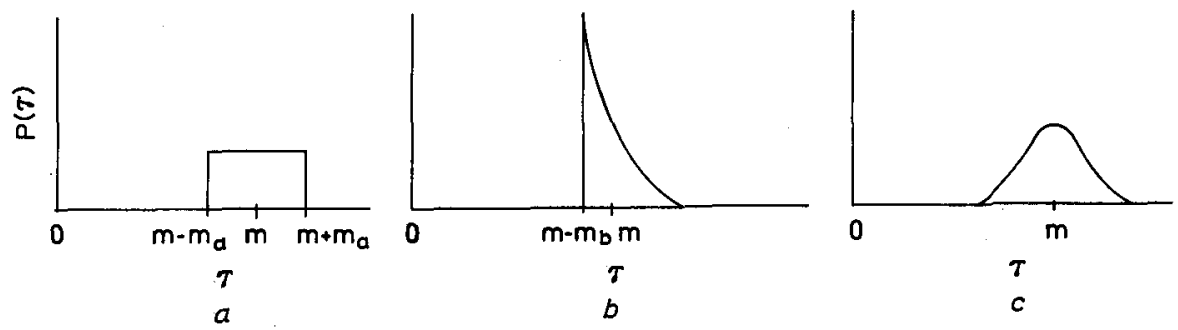

FIG. 1. Examples of stimulus-interval distributions $p_{1}(\tau)$.
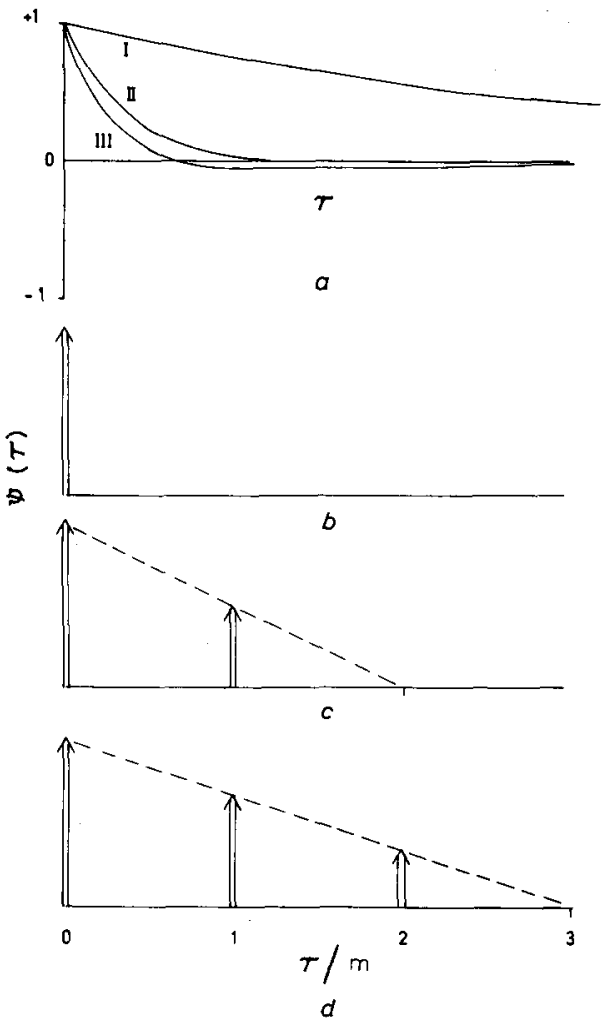

FIG. 2. Illustration of eqn. 1 for computation of variance of average response var $N$ in the case of periodic stimulation. Number of stimuli: $N=1,2$ and 3. In Fig. $2 a$ are drawn three noise autocorrelation functions. etc. It must be remarked, however, that low values of the ratio $f_{c} / f_{s}$ are of limited interest to cerebral evoked-potential averaging, when the alpha rhythm is taken as the interfering noise (and it is, moreover, taken for granted that these phenomena are independent of each other). The alpha rhythm $f_{c}$ is of the order of $10 \mathrm{~Hz}$. The stimulus-interval duration $m$ cannot be much smaller than $0.5 \mathrm{~s}$, as most evoked potentials have a duration of this order of magnitude. If $m$ is smaller than about $0.5 \mathrm{~s}$, subsequent evoked potentials will overlap and add linearly to each other, so that the initial component of the potential will be disturbed. In addition, there are indications that, for $m<0.5 \mathrm{~s}$, evoked potentials and the alpha rhythm interfere with each other in a nonlinear way (ARNAL and GERIN, 1969b; SATO et al, 1971). This implies that $m f_{c}=f_{c} / f_{s}>5$. Nevertheless, if $f_{c}$ is not stable from experiment to experiment, a fluctuation in the stimulus intervals tends to equalise the variances, which, as regards comparability, is a desirable feature.

The aim of the present communication is to clarify the foregoing comments and statements, and to elaborate the pertinent theory not only from $m f_{c}=n, n$ being a whole number, but also for $m f_{c}$ being a fractional number, including 
$m f_{c}=n+\frac{1}{2}$. Such an attempt may be helpful if one wants to decide whether the potential profits of aperiodic stimulation, in comparison with purely periodic stimulation, counterbalance the effort that must be made to obtain special stimulus conditions and to counteract the quality of high-precision devices. For the noise-power spectrum we have not taken a spectrum that is flat over a range $f_{x}$ and zero elsewhere, but spectra that are more realistic, at least as regards spectra

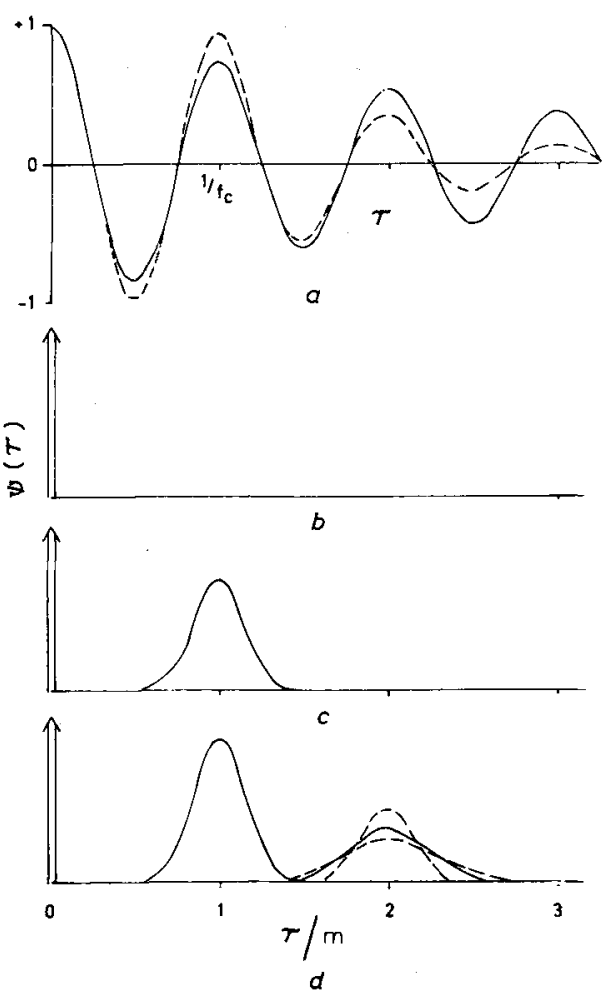

FIG. 3. Illustration of eqn. 2 for computation of variance of average response var $N$ in the case of aperiodic stimulation. Number of stimuli: $N=1,2$ and 3 . In Fig. $3 a$ two noise autocorrelation functions are drawn.

of biological origin. In terms of their autocorrelation functions

and

$$
\phi_{1}(\tau)=\exp (-\alpha|\tau|) \cos \left(2 \pi f_{c} \tau\right)
$$

$$
\phi_{2}(\tau)=\exp \left(-\alpha^{2} \tau^{2} / 2\right) \cos \left(2 \pi f_{c} \tau\right),
$$

will be investigated. The stimulus-interval distribution is also different from Ruchlin's; it is a Gaussian distribution $p_{1}(\tau)$, with mean $m$ and variance $\sigma_{1}^{2}$; it is shown in Fig. 1c. In practice, the latter distribution is probably easier to obtain from a wideband noise generator in combination with a relaxation oscillator.

Both modifications, the shape of the noise spectrum and the shape of the interstimulus interval, are inviting from a theoretical point of view, because the expressions for the variance of the averaged noise samples as a function of $N$ can be written in a closed mathematical form. Besides the situation that $m f_{c}=5$, relevant to e.e.g. applications as indicated, the result for $m f_{c}$ being about unity has also been treated, mainly because, under these circumstances, pecularities come to light which so far had not been reported: $\operatorname{var} N$ of the noise samples does not always decrease monotonically with increasing $N$.

Up to now it has been tacitly assumed that the aperiodicity in stimulation is of the renewal type; that is, the durations of successive intervals are independent of each other. We have taken the opportunity to investigate the outcome if this is not so, and when the interval durations are correlated with each other according to the Markov process. Here, again, we may conveniently use a Gaussian interval distribution.

\section{THEORY}

According to LEE (1960), the normalised variance of $N$ sample amplitudes relative to the value for $N=1$, for a fixed sample-time interval, is equal to

$$
\operatorname{var} N=N^{-1}\left\{1+2 \sum_{k=1}^{N-1}(1-k / N) \phi(k m)\right\}
$$

where $\phi(\tau)$ is the normalised autocorrelation function of the noise, $\phi(0)=1$ and $\phi(\infty)=0$.

This formula can be generalised when the sampling time interval is not constant. If the sampling-interval durations are stochastical distributed with a probability-density function $p_{1}(\tau)$ with mean $m$ and variance $\sigma_{1}{ }^{2}$, and with a probability-density function of the duration of the sum of $k$ successive intervals $p_{k}(\tau)$ with 
variance $\sigma_{k}^{2}$, the normalised variance is equal to

$$
\begin{aligned}
\operatorname{var} N=N^{-1}\left\{1+\sum_{k=1}^{N-1}(1-k / N)\right. \\
\left.\qquad \int_{-\infty}^{\infty} p_{k}(\tau) \phi(\tau) d \tau\right\}
\end{aligned}
$$

where $p_{k}(\tau)=p_{k}(-\tau)$ for $\tau<0$.

If the interval durations are independent of each other, it follows that

$$
p_{k}(\tau)=\int_{0}^{\tau} p_{k-1}(t) p_{1}(\tau-t) d t \text { for } k \geqslant 2
$$

For regular stimulation, $p_{k}(\tau)$ degenerates to a delta function: $p_{k}(\tau)=\delta(\mathrm{km})$.

To fix ideas, the expression in brackets in eqn. 1 is shown in Fig. 2 for $N=1,2$ and 3, and for $\tau>0$. $\operatorname{Var} N$ is equal to the weighted sum of the values of $\phi(\tau)$ at $\tau=0, m$ and $2 m$. For

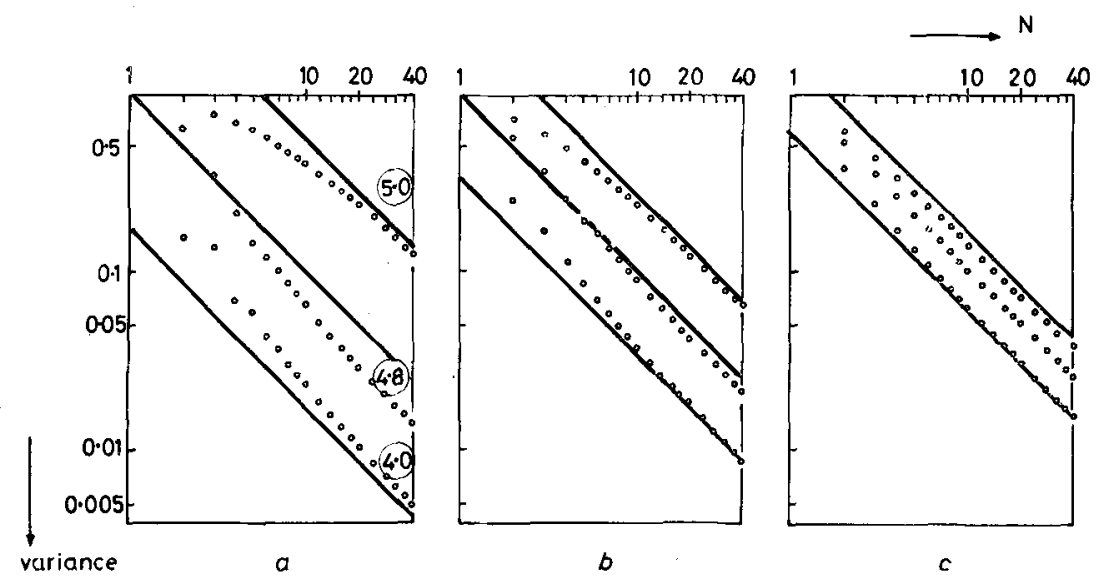

FIG, 4. Normalised variance of average response var $N$ for noise autocorrelation function

$$
\phi_{1}(\tau)=\exp (-\alpha|\tau|) \cos 2 \pi f_{c} \tau .
$$

In Figs. $4 a, b$ and $c$, the value of $\alpha / f_{c}$ increases from $0 \cdot 314 / 4$ to $0 \cdot 314 / 2$ and $0 \cdot 314$. Inset numbers refer to the value of $m f_{c}$. Periodic stimulation with interval duration $m$.

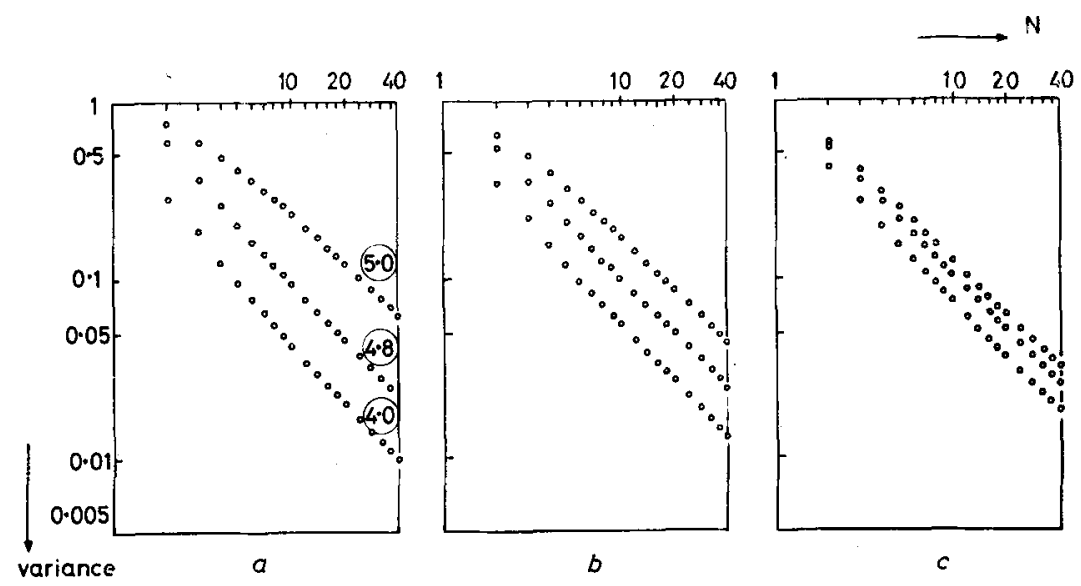

FIG. 5. As Fig. 4 for aperiodic stimulation, viz. Gaussian-distributed stimulus-interval durations with mean $m$ and standard deviation $\sigma_{1} ; \sigma_{1} / m=0.15$. 
$\phi(\tau)=\exp (-\alpha \tau)$, cf: curves (i) and (ii), one has, respectively, for the said values of $N$, and

$$
\operatorname{var} N=1,\left\{1+2 \cdot \frac{1}{2} \exp (-\alpha m)\right\} / 2
$$

$$
\{1+2 \cdot 2 / 3 \exp (-\alpha m)+2 \cdot 1 / 3 \exp (-2 \alpha m)\} / 3 .
$$

If $\alpha$ is smaller than $1 / m$, cf. curve (i), $\operatorname{var} N$ will be larger than if the reverse holds. If $\alpha$ is much larger than $1 / m$, cf. curve (ii), the expressions in brackets are nearly equal to unity. For bandfiltered Gaussian noise, cf. curve (iii), the higher-
Gaussian-Markov process, it follows that $\left(\sigma_{k} / \sigma_{1}\right)^{2}=k+2 \rho\left(\rho^{k}-k \rho+k-1\right)(1-\rho)^{-2}$. The dashed curves in Fig. $3 d$ are valid for $\rho=+0.5$ and $\rho<-0.5$, so that $\sigma_{2}^{2}=3{\sigma_{1}}^{2}$ and $\sigma_{2}{ }^{2}=\sigma_{1}{ }^{2}$.

For ease of computation, and without loss of generality, we have assumed in the following that $3 \sigma_{1}+m$. A more severe restriction on $\sigma_{1}$ is set by the requirement that the observation time of the evoked potential, which may be set equal to $m-3 \sigma_{1}$, shall not be much smaller than $m$,

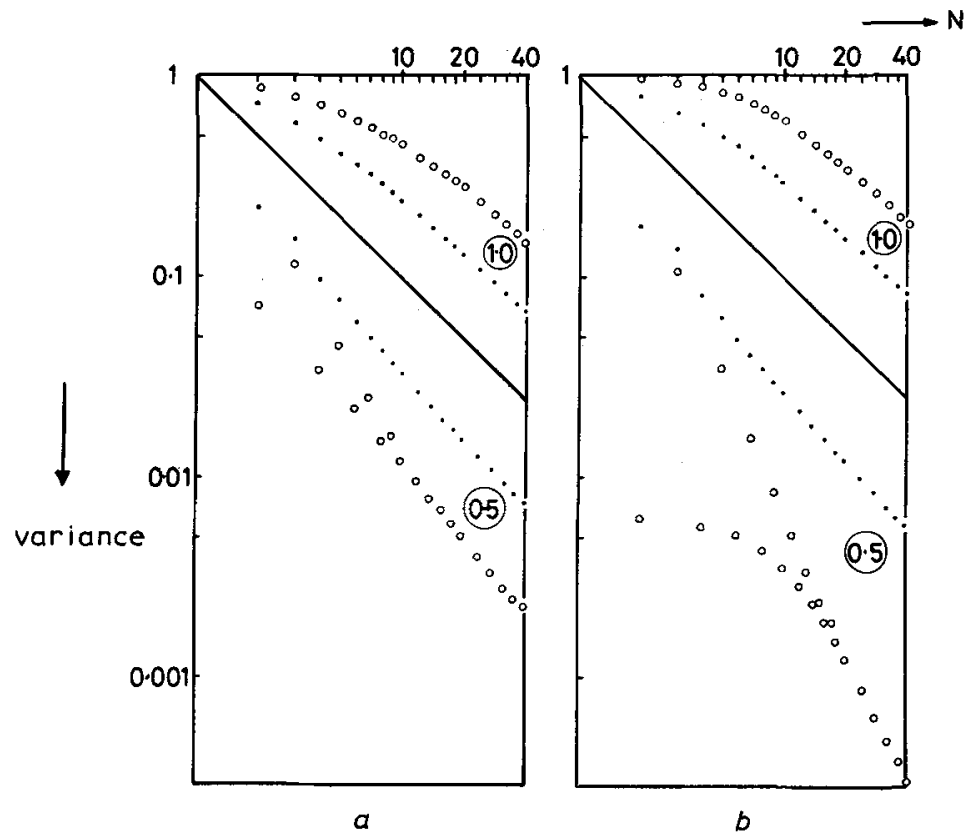

FIG. 6. Normalised variance of average response var $N$. In Fig. $6 a$, the noise autocorrelation $=\phi_{1}(\tau)=\exp (-\alpha|\tau|) \cos 2 \pi f_{c} \tau$, and in Fig. $6 b$ $\phi_{2}(\tau)=\exp \left(-\alpha^{2} \tau^{2} / 2\right) \cos 2 \pi f_{c} \tau$; in both cases $\alpha / f_{c}=0 \cdot 314$. Inset numbers refer to the value of $m f_{c}$. Open circles for $\sigma_{1} / \mathrm{m}=0$, solid circles for $\sigma_{1} / m=0 \cdot 15$.

order contribution to $\operatorname{var} N$ is negative, though not much so in the underlying case.

Fig. 3 is intended to clarify eqn. 2 , which again applies to $N=1,2$ and 3 . The solid curve in Fig. $3 a$ represents $\phi_{1}(\tau)$, the dashed curve $\phi_{2}(\tau)$, both with $\alpha=0.314 f_{c}$. For $p_{1}(\tau)$ one writes $p_{1}(\tau)=\exp \left\{-(m-\tau)^{2} /\left(2 \sigma_{1}^{2}\right)\right\} \quad\left(\sigma_{1} \sqrt{ } 2 \pi\right)^{-1}$. It has been taken that $\sigma_{1}=0.15 \mathrm{~m}$ and $m=1 / f_{c}$. The distribution $p_{2}(\tau)$ has a similar shape with mean $2 m$ and variance $\sigma_{2}{ }^{2}$. If the intervals are independent of each other, then $\sigma_{2}{ }^{2}=2 \sigma_{1}{ }^{2}$. If the intervals are distributed according to a i.e. the time period available for periodic stimulation.

Expressions for variance may be given, if required, in the frequency domain, by substituting

and

$$
\phi(f)=\int_{-\infty}^{\infty} \phi(\tau) \exp (-2 \pi j f \tau) d \tau
$$

$$
P_{k}(f)=\int_{-\infty}^{\infty} p_{k}(\tau) \exp (2 \pi j f \tau) d \tau
$$


Then the normalised variance equals

$$
\begin{aligned}
\operatorname{var} N=N^{-1}\left\{1+\sum_{k=1}^{N-1}(1-k / N)\right. \\
\\
\left.\int_{-\infty}^{\infty} \phi(f) P_{k}(f) d f\right\}
\end{aligned}
$$

For mutually independent interval durations, one inserts $P_{k}(f)=P_{1}^{k}(f)$. Substituting

$$
\phi_{1}(\tau)=\exp (-\alpha|\tau|) \cos \left(2 \pi f_{c} \tau\right)
$$

and

$$
p_{k}(\tau)=\exp \left\{-(k m-\tau)^{2} /\left(2 \sigma_{k}^{2}\right)\right\}\left(\sigma_{k} \sqrt{ }(2 \pi)^{-1}\right.
$$

in eqn. 2 , or

$$
\begin{aligned}
\phi(f)= & 2 \alpha\left\{(2 \pi f)^{2}+\alpha^{2}+\left(2 \pi f_{c}\right)^{2}\right\} \\
& \times\left[\left\{(2 \pi J)^{2}-\alpha^{2}-\left(2 \pi f_{c}\right)^{2}\right\}^{2}+4 \alpha^{2}(2 \pi f)^{2}\right]^{-1}
\end{aligned}
$$

and

$$
P_{k}(f)=2 \exp \left(-2 \pi^{2} f^{2}{\sigma_{k}}^{2}\right) \cos (2 \pi f k m)
$$

in eqn. 3 yields

$$
\begin{aligned}
\operatorname{var} N= & N^{-1}\left[1+2 \sum_{k=1}^{N-1}(1-k / N) \cos \left(2 \pi f_{c} m_{k}{ }^{\prime}\right)\right. \\
& \left.\quad \exp \left\{-4 \pi^{2} f_{c}{ }^{2} \sigma_{k}{ }^{2} / 2-\alpha k m+\alpha^{2}{\sigma_{k}}^{2}{ }_{2}\right\}\right]
\end{aligned}
$$

where $m_{k}{ }^{\prime}=k m-\alpha \sigma_{k}{ }^{2}$, and provided $m_{k}{ }^{\prime}>3 \sigma_{k}$ With $\phi_{2}(\tau)=\exp \left(-\alpha^{2} \tau^{2} / 2\right) \cos \left(2 \pi f_{c} \tau\right)$, or

$$
\phi(f)=\sqrt{ }(\pi / 2) x^{-1}\left\{\exp -2 \pi^{2}\left(|f|-f_{c}\right)^{2} \alpha^{-2}\right\}
$$

one finds

$$
\begin{aligned}
\operatorname{var} N=N^{-1} & {\left[1+2 \sum_{k=1}^{N-1}(1-k / N)\left(1+\alpha^{2} \sigma_{k}^{2}\right)^{-0.5}\right.} \\
& \times \cos \left(2 \pi f_{c} m_{k}{ }^{\prime \prime}\right) \exp \left\{-\left(2 \pi^{2} f_{c}^{2} \sigma_{k}^{2}\right.\right. \\
& \left.\left.+\alpha k m / 2)\left(1+\alpha^{2} \sigma_{k}^{2}\right)^{-1}\right\}\right]
\end{aligned}
$$

$m_{k}^{\prime \prime}=k m\left(1+\alpha^{2}{\sigma_{k}}^{2}\right)^{-1}$ provided $m_{k}^{\prime \prime}>3 \sigma_{k}$.

\section{RESULTS}

Figs. 4 and 5 show, on a double-logarithmic scale, the relationship between the normalised variance, shortened to $\operatorname{var} N$, as a function of the number of stimulations $N$. The noise has a correlation function $\phi_{1}(\tau)$, with $\alpha / f_{c}=0 \cdot 314 / 4$, $0.314 / 2$ and 0.314 in this order, from left to right. This amounts to a bandwidth, defined as the frequency range at half the maximum value in the power spectrum, of approximately $f_{x} / f_{c}=0 \cdot 1 / 4,0 \cdot 1 / 2$ and $0 \cdot 1$, respectively. The latter two values compare roughly to the bandwidth of the alpha rhythm. Note that the maximum-value frequency in the power spectrum does not coincide exactly with $f_{c}$, but is a few per cent smaller at the values of $\alpha$ used.

The results for regular stimulation, with $\sigma_{1}=0$, are computed and they are shown in Figs. $4 a, b$ and $c$, and those for Gaussiandistributed intervals with $\sigma_{1} / m=0.15$ are shown in Figs. $5 a, b$ and $c$, for $m=5 / f_{c}$ (upper set of points), $m=4 \cdot 8 / f_{c}$ and $m=4 \cdot 5 / f_{c}$ (lower set of points). If $f_{c}=10 \mathrm{~Hz}$, this corresponds to stimulation frequencies $f_{s}=2,2 \frac{1}{12}$ and $2 \frac{2}{9} \mathrm{~Hz}$. It will be observed that a considerable difference exists in the values of the variance for $m=5 / f_{c}$ and $m=4 \cdot 5 / f_{c}$, and this the more so when the noise is narrower in bandwidth $(\alpha$ smaller). For $\sigma_{1}=0$, the asymptotes for large values of $N$ can readily be determined from the expression

$\operatorname{var} N=N^{-1}\{\exp (2 \alpha m)-1\}\{\exp (2 \alpha m)$

$$
\left.-2 \exp (\alpha m) \cos \left(2 \pi f_{c} m\right)+1\right\}^{-1}
$$

In Fig. 4 , these have been drawn for $m f_{c}=4.5$ and 5 .

From the above formulas for the asymptotes, it follows that, for $\sigma_{1}=0, \operatorname{var} N$ converges to $1 / N$ when the noise becomes broader in bandwidth ( $\alpha$ larger). For $\sigma_{1} \neq 0$, the same conclusion would seem to hold at first sight, but on second thoughts this is not true. This cannot be deduced from the formulas given so far because these are valid for $k m_{k}-\alpha \sigma_{k}^{2}>3 \sigma_{1}$. For large values of $\alpha$, the autocorrelation is practically equal to $\phi(\tau)=\exp (-\alpha \tau)$. From eqn. 2, one finds that

$$
\begin{aligned}
\operatorname{var} N & =N^{-1}\left\{1+2(1-1 / N) \exp \left(-\alpha m+\alpha^{2} \sigma^{2}{ }_{1} / 2\right)\right. \\
& \left.+2(1-2 / N) \exp \left(-2 \alpha m+2 \alpha^{2} \sigma_{1}{ }^{2} / 2\right)+\ldots\right\}
\end{aligned}
$$


Since each term in this expression is equal to, or larger than, the corresponding terms in the similar expression with $\sigma_{1}=0$, for very broadband noise the variance is larger in the case of aperiodic stimulation than for periodic stimulation, whatever the values of $N$ or $m f_{c}$. The intriguing question remains whether this statement may be generalised to other than Gaussiandistributed interstimulus intervals.

For $m f_{c}=4 \cdot 5$, the normalised variance, as a function of $N$, does not change as smoothly as for $m f_{c}=5$, especially for small $\alpha$, small $\sigma_{1}$ and small $N$ values. The effect becomes obscured when the numerical values of these parameters increase. The phenomenon is very pronounced for $m f_{c}=0 \cdot 5$. This is shown in Fig. 6 for $\sigma_{1} / m=0$ (open circles) and $\sigma_{1} / m=0.15$ (solid circles); the upper points refer to $m f_{c}=1$ and the lower points to $m f_{c}=0 \cdot 5$. Fig. $6 a$ holds for an autocorrelation function $\phi_{1}(\tau)$ and Fig. $6 b$ for $\phi_{2}(\tau)$, both with $\alpha / f_{c}=0 \cdot 314$, so that the power spectra have nearly the same bandwidth $f_{x}=0 \cdot 1 f_{c}$. The remarkable conclusion is that, under certain conditions, it is profitable, with regard to the variance, to utilise $N$ samples instead of $N+1$ samples.
If the stimulation intervals are not distributed according to renewal process, functions $p_{k}(\tau)$ for $k \geqslant 2$ are not determined solely by $p_{1}(\tau)$ but also by the nature of the interdependence of successive interval durations. Computations have been carried out for $\phi_{1}(\tau)$ with $\alpha / f_{c}=0.314$ and stimulus-interval durations following a Gaussian-Markov process with parameter $\rho$. The influence of $\rho$ is more evident with smaller values of $\alpha$ and $\sigma$. Fig. 7 shows the results for $\rho=-0.5$ (open circles), $\rho=+0.5$ (solid circles) and $\sigma_{1} / m=0 \cdot 5$. In Fig. $7 a$, the lower pair of figures holds for $m f_{c}=0.5$ and the upper pair for $m f_{c}=1$. Fig. $7 b$ holds for $m f_{c}=0 \cdot 9$, and Fig. $7 c$ for $m f_{c}=1 \cdot 2$. For $\rho=0$, the values for the normalised variance are for any $N$ situated between the two given sets of points.

The effect of correlation is relatively small, and will not be discussed further. Moreover, positive correlation sometimes results in a smaller variance than negative correlation; at other times the reverse holds.

\section{DISCUSSION}

Throughout this paper, we have assumed that the response wave is deterministic, and that the

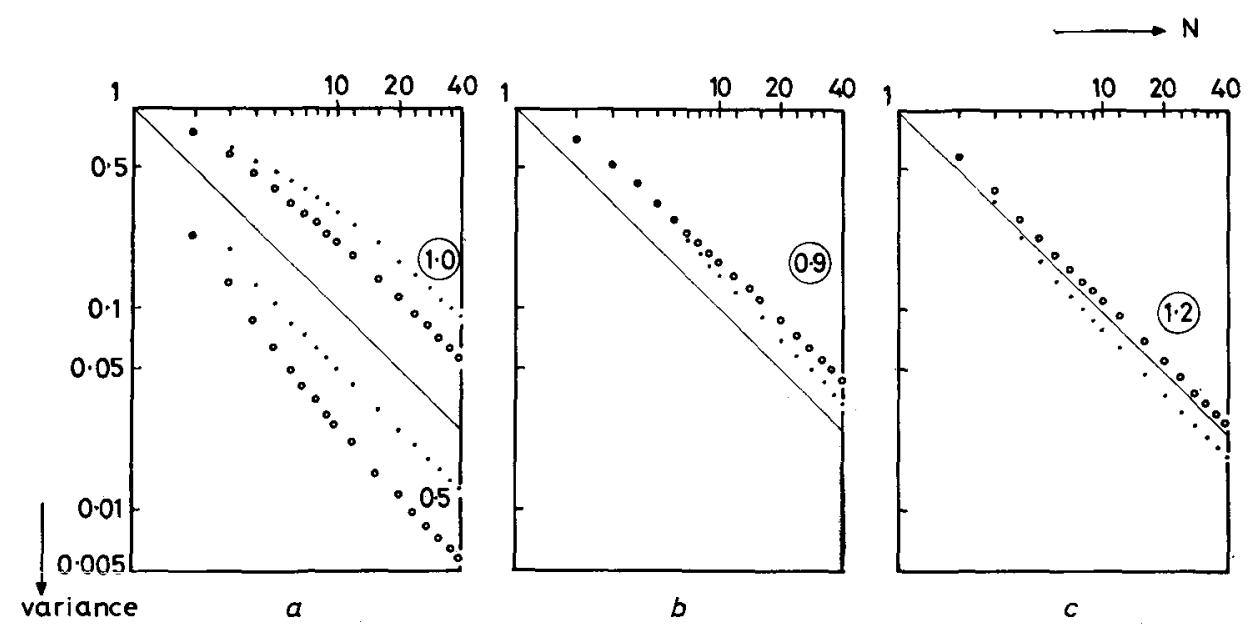

FIG. 7. Normalised variance of average response var $N$, for aperiodic stimulation with $\sigma_{1} / m=0 \cdot 15$; stimulus interval durations correlated according to a Gaussian-Markov process with $\rho=+0.5$ (open circles) and $\rho=-0.5$ (solid circles). Inset numbers refer to the value of $m f_{c}$. 
stationary background noise is additive to, and not time-locked to, the response. Each of these assumptions is open to doubt. If the response is nondeterministic, and if its random aspects are uncorrelated with the noise, then the total variance of the average response is equal to the sum of the variances caused by each of these sources of randomness. BARLow (1967) has carried out electronic simulations of the phenomenon of rhythmic after-discharge in the e.e.g. induced by photic stimulation, and he has investigated the influence of variable amplitude, latency, and a combination of both, in the evoked response. Further, a nonlinear interaction between the photically evoked response and the background activity was indicated. The influence of nonstationary noise is, in general, difficult to assess. Examples of nonstationary noise with regard to average-response computation have been given by BENDAT (1964).

In the computations, some properties of the alpha rhythm have been inserted for those of the interfering noise. The autocorrelation function of the alpha rhythm is mostly of a damped oscillatory type, and is often expressed as $\phi_{1}(\tau)=\exp (-\alpha \tau) \cos 2 \pi f_{c} \tau$. Although this rhythmicity has been subjected extensively to quantitative analysis, proper values for $\alpha$ are not easy to obtain from the literature.

SATo (1957) deduced from an occipital e.e.g. of a normal adult, that $\alpha=12.17 \mathrm{rad} / \mathrm{s}$, or $\alpha=12 \cdot 17 /(2 \pi)=1.93 \mathrm{~Hz}$. For $f_{c}=10 \mathrm{~Hz}$ one has $\alpha / f_{c}=0 \cdot 193$. WeIss (1959) has compared the e.e.g. with bandlimited noise and inserted in the above formula for the autocorrelation function $\alpha / f_{c}=0 \cdot 175$. DANIEL (1965) carried out measurements on the autocorrelogram of $1 \mathrm{~min}$ recordings from the occipital lobe. From his synchronisation ratio, one arrives at values for $\alpha / f_{c}$ ranging from 0.25 to 0.40 , in the eyesclosed condition. Recently, WENNBERG and ZETTERBERG (1971) studied quantitatively the shape of the autocorrelation functions of the e.e.g. which were thought to be composed of more than one component. As far as the alpha rhythm in the two occipital leads is concerned, one derives from their table for $\alpha / f_{c}$ (in our nomenclature) values of $0 \cdot 22$ to $0 \cdot 30$. These values are of the same order of magnitude as those used by us. It implies that the autocorrelation function, after a delay equal to about five periods, has diminished to roughly half of the maximum value at zero delay. Similar pictures result if one simulates the normal e.e.g. as the output of a tuned circuit fed by randomly occurring pulses as input (BARLow, 1962).

From the results, it can be deduced that, for given noise characteristics ( $\alpha$ and $f_{c}$ ) and a given stimulus frequency, the introduction of a fluctuation $\left(\sigma_{1}\right)$ in the stimulus-interval duration has the same effect on the variance of the average as if one were using regular stimulation, while the noise would have a more broadband power spectrum. This was also found experimentally by ARNAL and GERIN (1969a) for both the alpha rhythm and an electronically simulated version of it.

To be more specific, if the stimulus frequency $f_{s}$ is such that $f_{c} / f_{s}$ is approximately equal to a whole number $n$, on increasing the degree of fluctuation, the variance of the average decreases towards the value of $1 / N$, when $N$ is the number of stimuli presented. This has also been derived by RuCHLIN (1965) for other stimulus-interval distributions and for a different noise-power spectrum. Therefore, under these circumstances, aperiodicity in the stimulus-sequence presentation is advantageous. The value of this depends on the parameter values proper. However, if $f_{c} / f_{s}$ is equal to about $n+\frac{1}{2}$, the variance of the average tends to increase towards the value $1 / N$; aperiodicity worsens the result.

When the noise parameters $\alpha$ and $f_{c}$ and the stimulation frequency $f_{s}$ are such that it is possible to approximate to the condition $\operatorname{var} N=1 / N$ through the application of aperiodic stimulation and at the expense of a shorter effective observation time for the response wave, it is still not self-evidently advisable to use such a procedure of stimulation.

If the condition that $m f_{c}=n+\frac{1}{2}$ can be achieved, the variance can be diminished in an easier way. For instance, if $f_{c}=10 \mathrm{~Hz}$, an interstimulus interval of $m=1.05$ or $0.95 \mathrm{~s}$ will 
make $\operatorname{var} N$ smaller than $1 / N$, the value that, with the same mean interval duration, may ultimately be achieved for highly aperiodic stimulation.

The influence of aperiodicity on the variance largely depends on the value of $\alpha$, for a given ratio of $f_{s}$ and $f_{c}$. It may be asked how far these statements are relevant to average-response computation when the alpha rhythm stands for the disturbing noise component. In this respect, the outcome is fairly negative. Taking the parameter values as in Figs. $4 c$ and $5 c$, $\alpha / f_{c}=0.314, \quad f_{c}=10 \mathrm{~Hz}$ and $f_{s}=2.0 \mathrm{~Hz}$ $\left(m f_{c}=5\right)$, then for $N=10$ and $\sigma_{1} / m=0 \cdot 15$, the variance amounts to a value that is only $16 \%$ smaller in comparison with strictly periodic stimulation, where $\sigma_{1} / m=0$. Similarly, for $f_{s}=2 \cdot 2 \mathrm{~Hz} \quad\left(m f_{c}=4 \cdot 5\right)$, the variance is $17 \%$ larger than for $\sigma_{1} / m=0$. For larger values of $N$ and slower stimulation frequencies, the percentages become even smaller. Only in the case of noise with a very narrow power spectrum does aperiodic stimulation have real advantages. The alpha rhythm would seem to possess such a degree of irregularity that the reduction in observation time of the response wave and the requisites of unusual stimulation procedures are hardly outweighed by the eventual advantages.

A similar statement has been reached by KITSADO and HATSUDA (1965) when they reported and discussed experiments to determine the degree of contamination of the average response with alpha waves and the factors influencing the degree of contamination. Artificial signals, with the same amplitude and zero-crossings distribution as an e.e.g. filtered through an alpha bandpass filter, obtained by manually changing the frequency of an oscillator, were summed. Because the amplitude of the summed waves increased in the same way as sine waves if summed aperiodically, it was concluded that the frequency of ordinary alpha waves is random enough to be treated in the same manner as sine waves summed with random phase differences.

A direct application of the theory has been demonstrated by BALL et al. (1971), in an attempt to determine sensory-conduction veloci- ties by using evoked somatosensory responses recorded through the intact scalp as the latency indicators after stimulation of nerves in the periphery at distal and proximal points. These authors pointed out that, since the stimulus pulse from the computer was locked to the $60 \mathrm{~Hz}$ mains frequency, any $60 \mathrm{~Hz}$ artefact time-locked with the stimulus appeared as a signal, not as noise. To overcome this, the computer program randomly varied the $2 \mathrm{~s}$ triggering interval of the stimulation by $\pm 8.33 \mathrm{~ms}$, a range equal to the period of a $60 \mathrm{~Hz}$ frequency. The stimulus thus lost its time-lock to that frequency, and this artefact was averaged out.

Variation in the stimulation period has also been utilised in a slightly different context by MARSONER and TATSUNo (1970) in a study on evoked potentials triggered by a certain component of the background activity. In their setup, the sweep of the averager for evoked potentials is triggered when the alpha component of the e.e.g. rises above a certain level. In this case, the averaging cycle is phase-locked to the alpha wave, thus essentially influencing the result. For this reason, these authors use a random trigger delay between an alpha wave with sufficient amplitude and the triggering of the summation period.

\section{REFERENCES}

Arnal, D. and Gerin, P. (1969a) Etude du bruit résiduel des potentiels évoqués moyens. Electroencephal. Clin. Neurophysiol. 27, 315-321

ARnal, D. and Gerin, P. (1969b) Comparaison des potentiels évoqués moyens à l'aide d'un test $T^{2}$ généralisé. Application au problème de l'interférence des réponses évoqués visuelles successives. Electroencephal. Clin. Neurophysiol. 26, 325-331.

Ball, G. J., Saunders, M. S. and Schnabl, J. (1971)

Determination of peripheral sensory nerve conduction velocities in man from stimulus response delays of the cortical evoked potentials. Electroencephal. Clin. Neurophysiol. 30, 409-414.

BARLOW, J. S. (1962) Simulation of normal and abnormal electroencephalograms. Quart. Prog. Rept. 65, Res. Lab. Electron., Massachusetts Institute of Technology, Cambridge, Mass., April 15, 221-227.

BARLOW, J. S. (1967) Electronic simulation as an aid in evaluating computer-analysed EEG data. Electroencephal. Clin. Neurophysiol. 22, 381-386.

BENDAT, J. S. (1964) Mathematical analysis of average response values for non-stationary data. IEEE Trans. BME-11, 72-81.

DANIEL, R, S. (1965) Electroencephalographic correlogram ratios and their stability. Science, 145, 721-723. 
Kitasato, H. and Hatsuda, T. (1965) The degree of contamination of averaged response with alpha waves and alpha-like waves evoked by photic stimulation. Jap. J. Physiol. 15, 492-504.

LEE, Y. W. (1960) Statistical theory of communication (Wiley), 284.

Marsoner, H. J. and TAtsuno, J. (1970) A method for triggering evoked potentials by a certain component of the background activity. Med. \&Biol. Eng. 8, 415-418.

SATO, K. (1957) An interpretation concerning physiological significance of statistical nature of electroencephalograms. Folio Psychiatrica et Neurologica Japonica 10, 283-294.
Sato, K., Kitajima, H., Mimura, K., Hirota, N., TAgaWA, Y., and OCHI, N. (1971) Cerebral visual evoked potentials in relation to EEG. Electroencephal. Clin. Neurophysiol. 30, 123-138.

RuCHLIN, D. S. (1965) An analysis of average response computations based upon aperiodic stimuli. IEEE Trans. BME-12, 87-94.

WeIss, T. F. (1959) In Processing neuroelectric data, technical report 351, Res. Lab. Electronics, Massachusetts Institute of Technology, Cambridge, Mass.

Wennberg, A. and ZetTerberg, L. H. (1971) Application of a computer-based model for EEG analysis. Electroencephal. Clin. Neurophysiol. 31, 457-468

\section{ASPECTS DU CALCUL DU POTENTIEL EVOQUE MOYEN PAR STIMULATION APERIODIC}

Sommaire-Une analyse mathématique de la variance du potential évoqué moyen comme fonction du nombre de stimulations présentées, $N$, est dérivée, sous condition que la réponse soit perturbée additivement par bruit de fond stationnaire. On compare la variance en cas de stimulation strictement périodique, et en case de stimulation aléatoire, c'est à dire, si les intervalles entre les stimulations varient selon une distribution Gaussienne. En outre, les intervalles peuvent être corrélés mutuellement, par example conformément un processus Markov-Gaussien. Il est déduit que par l'introduction d'une stimulation apériodique, en général, la relation entre la variance et $N$ s'incline vers le cas d'un bruit de spectre à large bande, donc la variance ètant proportionale à $1 / N$.

\section{ASPEKTE DER MITTELUNG VON EVOZIERTEN REAKTIONS-POTENTIALE BEI APERIODISCHER STIMULATION}

Zusammenfassung-Eine mathematische Analyse der Varianz des gemittelten evozierten Reaktions-potentiales als Funktion der Anzahl von angebotene Reize $N$ wird dargestellt, unter der Voraussetzung dass der evozierte Potential von additiven stationären Rauschen gestört wird. Es werden verglichen die Varianz wenn die Reize rein periodisch erfolgen und wenn die Zeitintervalle zwischen Reize normal verteilte Schwankungen unterworfen sind. Im letzten Falle dürfen die Zeitintervalle wechselseitig korreliert sein, z.B. gemäss einem Gausschen Markov Prozess. Es wird gezeigt dass im Allgemeinen bei der Einführung von aperiodische Reizfolgen das Funktionalverband zwischen Varianz und $N$ sich dem Falle zustrebt wenn Rauschen mit breitbandigem Leistungsspektrum vorliegt, wobei also die Varianz proportional zu $1 / N$ ist. 